\title{
Actividad sanguínea de superóxido dismutasa y glutatión peroxidasa en novillas a pastoreo
}

\author{
Santiago Jaramillo( ${ }^{(1)}$, Néstor Alonso Villa ${ }^{(1)}$, Andrés Felipe Pineda ${ }^{(1)}$, Ángela Beatriz Gallego ${ }^{(1)}$, \\ Paola Tabares $^{(1)}$ y Alejandro Ceballos ${ }^{(1)}$
}

(1)Universidad de Caldas, Dep. de Salud Animal, AA 275, Manizales, Colombia. E-mail: aleceballos@ucaldas.edu.co

\begin{abstract}
Resumen - El objetivo de este estudio fue determinar el balance metabólico nutricional de Cu, Zn, Fe, Mn y Se, mediante la actividad sanguínea de SOD y GSH-Px y establecer la relación entre la concentración de Cu, Fe, Mn y Zn en el forraje y la actividad de SOD. Se tomaron $10 \mathrm{~mL}$ de sangre a 105 novillas seleccionadas en 15 rebaños lecheros de Caldas, Colombia ( $5^{\circ} 4^{\prime} \mathrm{N}$ y $75^{\circ} 3^{\prime} \mathrm{O}$ ) y se tomaron muestras de forrajes para analizar $\mathrm{Cu}$, Fe, Mn y Zn. El promedio de la actividad de SOD fue $1.390 \pm 1.299 \mathrm{U} \mathrm{g}^{-1} \mathrm{Hb}$, y estaba correlacionada con $\mathrm{Cu}$, Mn y Fe en el forraje. La actividad promedio de GSH-Px fue $389 \pm 184 \mathrm{U} \mathrm{g}^{-1} \mathrm{Hb}$ y fue observada una mayor frecuencia de valores deficitarios y bajo/marginales de $9 \%$, habiendo sido más afectados los animales de zonas altas (>2.000 msnm). Bajo estas condiciones, estos resultados permiten señalar que la SOD es una enzima que puede emplearse como indicador del balance metabólico nutricional de $\mathrm{Cu}, \mathrm{Mn}$ y Fe en bovinos a pastoreo, no está clara todavía su relación con Zn. La actividad de GSH-Px indica deficiencias en el balance metabólico nutricional de Se, en bovinos a pastoreo.
\end{abstract}

Términos para indexación: bovinos, oligoelementos, antioxidantes, enzimas.

\section{Blood activity of superoxide dismutase and glutathione peroxidase in grazing dairy heifers}

\begin{abstract}
The objective of this study dealt with the evaluation of the metabolic nutritional status of $\mathrm{Cu}, \mathrm{Zn}$, $\mathrm{Mn}, \mathrm{Fe}$ and Se in grazing dairy heifers, through the determination of the activity of SOD and GSH-Px in blood. Blood samples $(10 \mathrm{~mL})$ were taken from 105 heifers randomly selected in 15 dairy farms in Caldas, Colombia $\left(5^{\circ} 4^{\prime} \mathrm{N}\right.$ y $75^{\circ} 3^{\prime} \mathrm{W}$ ). Forage samples were also collected to analyze $\mathrm{Cu}, \mathrm{Fe}, \mathrm{Mn}$, and $\mathrm{Zn}$. Mean activity of SOD was $1,390 \pm 1,299 \mathrm{U} \mathrm{g}^{-1} \mathrm{Hb}$, and it was related to the content of $\mathrm{Cu}, \mathrm{Mn}$, and Fe in the forage. Mean blood activity of GSH-Px was $389 \pm 184 \mathrm{U} \mathrm{g}^{-1} \mathrm{Hb}$. The highest frequency of values under the reference was about $9 \%$; animals located over 2,000 $\mathrm{m}$ over sea level were the most affected. SOD is useful to determine the nutritional metabolic status of $\mathrm{Cu}, \mathrm{Mn}$ and $\mathrm{Fe}$, but the relationship with $\mathrm{Zn}$ is not clear yet, and requires further investigations. The blood activity of GSH-Px in grazing animals, was under the reference values, indicating Se deficiencies.
\end{abstract}

Index terms: bovine, oligoelements, antioxidants, enzymes.

\section{Introducción}

Las especies reactivas de oxígeno (ERO) son generadas en procesos metabólicos normales y no son nocivas en todos los casos, ya que los antioxidantes son la respuesta fisiológica de defensa contra los ERO (Miller et al., 1993). El anión superóxido y el peróxido de hidrógeno son algunos ejemplos de ERO relacionados con diferentes respuestas orgánicas y son metabolizados por algunas enzimas antioxidantes (Miller et al., 1993; Murray et al., 2000). Un desequilibrio entre la producción de ERO y los antioxidantes puede iniciar reacciones oxidativas en cadena y peroxidación lipídica que causan daños celulares graves (Miller et al., 1993; Campbell \& Miller, 1998; Murray et al., 2000).

En la célula se encuentran, entre otras, las enzimas antioxidantes superóxido dismutasa (SOD, EC 1.15.1.1) y glutatión peroxidasa (GSH-Px, EC 1.11.1.9) (Miller et al., 1993; Murray et al., 2000), encargadas de catalizar reacciones que reducen los ERO evitando los daños que 
causan estrés oxidativo (Miller et al., 1993). Los oligoelementos cobre $(\mathrm{Cu})$, hierro $(\mathrm{Fe})$, manganeso $(\mathrm{Mn})$ y zinc (Zn) forman parte de la estructura proteica de la SOD, mientras que el selenio (Se) forma parte de la GSH-Px; ambas enzimas se encuentran en el citosol y su función es la reducción del anión superóxido y del peróxido de hidrógeno, respectivamente, brindando protección a la célula y al organismo (Miller et al., 1993; Murray et al., 2000).

La característica de ser metaloenzimas permite que la determinación de su actividad pueda ser utilizada como marcador biológico del estado metabólico nutricional de los minerales que forman su estructura (Hambidge, 2003), cuya actividad en bovinos fue descrita por Awadeh et al. (1998), Wichtel (1998), Wittwer et al. (2002); en equinos por Ceballos et al. (1998b); y en porcinos por Lauridsen et al. (1999). Pese a lo anterior, en bovinos no se ha señalado la actividad de SOD dependiente de Fe y Mn.

La deficiencia en el consumo de esos minerales o la presencia de factores que puedan interferir en su metabolismo, especialmente en bovinos mantenidos en pastoreo, ocasionaría una disminución de la actividad enzimática, asociada con baja ganancia de peso (Wichtel et al., 1996), disminución de la calidad de la carne (Lauridsen et al., 1999), casos de mastitis y aumento en el recuento de células somáticas (Scaletti et al., 2003), enfermedad del músculo blanco, retención de placenta, diarrea, abortos, partos prematuros y alta mortalidad neonatal (Miller et al., 1993; Kincaid, 1995; Mee \& Rogers, 1996; Wichtel, 1998), disminución en la eficiencia reproductiva (Dargatz \& Ross, 1996; Campbell \& Miller, 1998), depresión inmunológica (Xin et al., 1991; Awadeh et al., 1998; Scaletti et al., 2003), anemia, baja calidad seminal y disminución de grasa en la leche (Underwood \& Suttle, 1999).

La concentración de Se, Cu y Zn en los suelos y forrajes es variable en diferentes regiones del mundo; encuéntranse zonas con concentraciones deficitarias en la costa oeste de Estados Unidos y Canadá, amplias áreas en China y Australia, Nueva Zelanda, los países escandinavos, sur de Francia, la región balcánica, norte de Inglaterra y Escocia ( Dargatz \& Ross, 1996; Mee \& Rogers, 1996; Wichtel, 1998; Pavlata et al., 2002). Igualmente en Suramérica se han encontrado zonas con concentraciones deficitarias de Se en forrajes (Ceballos et al., 1999). En Colombia no hay información actualizada con respecto al contenido de oligoelementos en forrajes, pese a ello, se han observado concentraciones consideradas deficitarias en los pastos del país (Laredo \& Cuesta, 1987), probablemente como reflejo de las características de los suelos colombianos (Malagón et al., 1995).

El objetivo de este estudio fue determinar el balance metabólico nutricional de $\mathrm{Cu}, \mathrm{Fe}$, Mn, Zn y Se, mediante la actividad sanguínea de SOD y GSH-Px en bovinos en crecimiento (novillas) a pastoreo, en explotaciones lecheras de Caldas, Colombia, además de establecer la eventual relación entre la concentración de $\mathrm{Cu}, \mathrm{Fe}, \mathrm{Mn}$ y Zn en el forraje y la actividad sanguínea de SOD.

\section{Material y Métodos}

Se seleccionaron 15 explotaciones lecheras del suroccidente del departamento de Caldas, Colombia $\left(5^{\circ} 4^{\prime} \mathrm{N}\right.$ y $\left.75^{\circ} 3^{\prime} \mathrm{O}\right)$, teniéndose en cuenta su ubicación geográfica y clasificándolas como zona baja (ZB: $<1.000 \mathrm{msnm}$ ), zona media (ZM: entre $1.000 \mathrm{y}$ $2.000 \mathrm{msnm}$ ) y zona alta (ZA: $>2.000 \mathrm{msnm}$ ), tipo de producción, grado de tecnificación, que llevaron registros y que los animales no recibieron un suplemento mineral adicional a la sal mineralizada consumida, cuyo rango composicional está descrito en el Cuadro 1.

Las explotaciones seleccionadas fueron clasificadas como lechería especializada en sistema de pastoreo intensivo. La raza predominante fue Holstein y algunos mestizajes con Pardo Suizo, Gyr lechero y Brahman. En las diferentes explotaciones estudiadas, las gramíneas fueron la principal fuente de alimentación para los animales, habiéndose encontrado distintas variedades, según la ubicación geográfica de la explotación, como: Kikuyo (Pennisetum clandestinum), Falsapoa (Holcus lanatus) y Rye grass (Lolium sp.) en la zona alta; Estrella (Cynodon sp.), Brachiaria (Brachiaria sp.) y

Cuadro 1. Rango de la composición mineral de los suplementos utilizados en 15 explotaciones lecheras de Caldas, Colombia.

\begin{tabular}{lc}
\hline Mineral & Contenido de minerales (rango) \\
\hline $\mathrm{NaCl}(\%)$ & $28-41$ \\
$\mathrm{Ca}(\%)$ & $4-18$ \\
$\mathrm{P}(\%)$ & $4-8$ \\
$\mathrm{~S}(\%)$ & $2,5-5,0$ \\
$\mathrm{Mg}(\%)$ & $0,3-3,5$ \\
$\mathrm{Cu}(\%)$ & $0,1-0,6$ \\
$\mathrm{Co}(\mathrm{ppm})$ & $40-100$ \\
$\mathrm{Zn}(\mathrm{ppm})$ & $400-1.000$ \\
$\mathrm{Se}(\mathrm{ppm})$ & $0-40$ \\
$\mathrm{I}(\mathrm{ppm})$ & $100-300$ \\
$\mathrm{Mn}(\mathrm{ppm})$ & $0-0$ \\
$\mathrm{Fe}(\mathrm{ppm})$ & $0-0$ \\
$\mathrm{~F}(\mathrm{ppm})$ & $400-800$ \\
\hline
\end{tabular}


Guinea (Panicum maximum) en las zonas media y baja. El sistema de pastoreo era directo rotacional, suplementado con sales minerales (Cuadro 1) en una cantidad diaria entre 30 y $60 \mathrm{~g}$ por novilla; el agua se mantuvo a voluntad. En ninguna de las explotaciones se administraban alimentos concentrados a las novillas seleccionadas.

Entre los meses de mayo y julio de 2003, en cada explotación se escogieron al azar siete novillas, con una edad promedio inferior a dos años; a cada una se le extrajeron $10 \mathrm{~mL}$ de sangre con heparina, mediante venopunción coccígea empleandose tubos al vacío. Las muestras fueron refrigeradas y llevadas al Laboratorio de Patología Clínica de la Universidad de Caldas, en las 12 horas siguientes, para su análisis. También se tomaron muestras de forraje de las praderas, destinadas al consumo animal, el mismo día en que se obtuvo la sangre.

Se escogieron al azar 10 submuestras (100 g), de diferentes sitios, en los potreros que estaban listos para ser consumidos por las novillas; las muestras fueron cortadas a $10 \mathrm{~cm}$ del suelo. Posteriormente, se homogeneizaron y se obtuvieron $500 \mathrm{~g}$ que fueron inmediatamente almacenados en bolsa de papel y en un lapso de 12 horas remitidos al Laboratorio de Bromatología, de la Universidad de Caldas, para su análisis. Los resultados se presentan consolidados para cada zona geográfica.

Inicialmente, se determinó la concentración de hemoglobina utilizándose el método de cianometahemoglobina; posteriormente, se separaron los eritrocitos contenidos en $500 \mu \mathrm{L}$ de sangre, mediante centrifugaciones sucesivas a 3.000 rpm por 10 minutos, se hemolizaron con agua destilada a $4^{\circ} \mathrm{C}$ y se hizo una dilución 1:50 con diluyente Ransod, para determinarse la actividad de SOD, mediante un método colorimétrico. Para la actividad de GSH-Px, la sangre fue hemolizada con diluyente Ransel, en una proporción 1:41 y la actividad fue determinada por una técnica cinética descrita por Ceballos et al. (1998a). El resultado de ambas pruebas fue expresado en $\mathrm{U} \mathrm{g}^{-1} \mathrm{Hb}$. La concentración de $\mathrm{Cu}$, $\mathrm{Zn}, \mathrm{Fe}$ y $\mathrm{Mn}$ en el forraje se analizó mediante espectrofotometría de absorción atómica.

Los datos se presentan mediante la obtención de las estimadas promedio, desviación estándar, rango, coeficiente de variación e intervalo de confianza al 95\% (Daniel, 2002). La comparación entre las medias de los grupos se hizo con el análisis de varianza ANDEVA para GSH-Px, considerándose un solo factor, determinado por la zona geográfica donde se encontraba el predio (ZB, ZM o ZA) siguiéndose el modelo: $X_{\mathrm{ij}}=\mu+\tau_{\mathrm{j}}+\varepsilon_{\mathrm{ij}}$, donde $\mathrm{X}_{\mathrm{ij}}$ es la observación; $\mu$ es la media general; $\tau_{\mathrm{j}}$ es la media del efecto del factor zona geográfica; $\varepsilon_{\mathrm{ij}}$ es el error. Para la comparación de la actividad de SOD, entre las diferentes zonas geográficas, se empleó un método de análisis de medianas jerarquizado (método de Kruskal-Wallis), dado el tipo de distribución de los datos (Daniel, 2002). Para ambas comparaciones se fijó la significancia en $\mathrm{p}<0,05$. La eventual asociación, entre la concentración en forraje de Cu, Zn, Mn y Fe y la actividad sanguínea de SOD en las novillas, se obtuvo mediante una regresión lineal simple (Daniel, 2002). Se obtuvo la frecuencia de valores alterados para la actividad de GSH-Px, agrupándolos en deficitarios $\left(<60 \mathrm{U} \mathrm{g}^{-1} \mathrm{Hb}\right)$, bajos (60-100 $\mathrm{U} \mathrm{g}^{-1} \mathrm{Hb}$ ) o marginales (100-130 U g ${ }^{-1}$ Hb) (Ceballos et al., 1998a).

\section{Resultados y Discusión}

El promedio para la actividad de la SOD fue de $1.390 \pm 1.299 \mathrm{U} \mathrm{g}^{-1} \mathrm{Hb}$, con un rango entre $218 \mathrm{y}$ $10.848 \mathrm{U} \mathrm{g}^{-1} \mathrm{Hb}$ (Cuadro 2). Se encuentra una información escasa acerca de la actividad de SOD en bovinos, los datos referenciales para su actividad sanguínea son limitados y la técnica para determinar la actividad varía entre las publicaciones consultadas (Xin et al., 1991; Arthington et al., 1996). En otros estudios realizados con bovinos lecheros, en la misma zona geográfica, Ceballos et al. (2003) describen valores similares a los observados en este estudio, para la actividad sanguínea de SOD. En estudios realizados con otras especies como equinos (Ceballos et al., 1998b), la actividad de SOD encontrada es similar a la de este

Cuadro 2. Promedio, desviación estándar (DE), rango, intervalo de confianza (IC) y coeficiente de variación (CV), para la actividad sanguínea de superóxido dismutasa (SOD) y glutatión peroxidasa (GSH-Px), en novillas a pastoreo en 15 explotaciones lecheras de Caldas, Colombia.

\begin{tabular}{cclcr}
\hline Ubicación geográfica $^{(1)}$ & ${\text { Promedio } \pm \mathrm{DE}^{(2)}}^{\text {Rango }}$ & \multicolumn{1}{c}{$\mathrm{IC}$} & $\mathrm{CV}(\%)$ \\
\hline ZB & $1.090 \pm 588 \mathrm{a}$ & $312-2.891$ & $2.640-4.870$ & 54 \\
$\mathrm{ZM}$ & $1.661 \pm 1.902 \mathrm{~b}$ & $218-10.848$ & $4.410-6.880$ & 115 \\
$\mathrm{ZA}$ & $1.421 \pm 1.015 \mathrm{c}$ & $262-4.189$ & $1.070-1.170$ & 71 \\
\hline ZB & $470 \pm 141 \mathrm{a}$ & $65-783$ & $422-519$ & 30 \\
ZM & $418 \pm 203 \mathrm{a}$ & $65-936$ & $348-488$ & 49 \\
ZA & $279 \pm 149 \mathrm{~b}$ & $57-558$ & $228-330$ & 54 \\
\hline
\end{tabular}

1)ZB: <1.000 msnm; ZM: 1.000-2.000 msnm; ZA: >2.000 msnm. (2)Promedios seguidos de letras diferentes presentan diferencias al $5 \%$ de probabilidad, en la prueba de análisis de varianza. 
estudio, pero con coeficientes de variación descritos inferiores.

El coeficiente de variación para la actividad de SOD, en este estudio, fue más bajo dentro de los grupos que entre los grupos, lo que indica que su determinación sería útil para establecer alteraciones debidas a factores nutricionales, ya que variaciones elevadas se encuentran asociadas con factores no homeostáticos o fisiológicos, lo que refleja, en mejor forma, cambios en el manejo nutricional de los animales (Herdt, 2000). Además, la actividad de SOD depende del consumo de minerales como Cu y Zn (Underwood \& Suttle, 1999), lo que la convierte en un marcador biológico confiable, afectado por el consumo de estos minerales y hácela útil para conocerse el balance metabólico nutricional de ellos (Xin et al., 1991; Arthington et al., 1996; Hambidge, 2003). Lo anterior indica la necesidad de recolectarse mayores antecedentes, con respecto a los valores referenciales para la actividad de esta enzima en bovinos y correlacionarla con la concentración sérica de minerales como $\mathrm{Cu}, \mathrm{Fe}, \mathrm{Mn}$ y $\mathrm{Zn}$.

La concentración de minerales, observada en las muestras de forrajes, se presenta en el Cuadro 3. Algunas de las explotaciones presentaron valores inferiores al considerado como crítico para bovinos en pastoreo (Underwood \& Suttle, 1999) y similares a lo descrito para las mismas gramíneas en Colombia (Laredo \& Cuesta, 1987). El contenido de $\mathrm{Cu}$, Fe y Mn varió según la zona geográfica y además se correlacionaba con la actividad de SOD, en sangre de las novillas que

Cuadro 3. Promedio, desviación estándar (DE), rango y coeficiente de variación (CV), para la concentración de $\mathrm{Cu}, \mathrm{Fe}, \mathrm{Mn}$ y Zn, en el forraje de 15 explotaciones lecheras, agrupadas según su ubicación geográfica en Caldas, Colombia.

\begin{tabular}{cccc}
\hline Ubicación geográfica $^{(1)}$ & Promedio $\pm \mathrm{DE}$ & Rango & $\mathrm{CV}(\%)$ \\
ZB & $9,5 \pm 5,0$ & $\mathrm{Cu}$ \\
ZM & $9,5 \pm 3,5$ & $5,1-19,5$ & 60 \\
ZA & $24,7 \pm 4,7$ & $5,4-81,0$ & 37 \\
ZB & $\mathrm{Fe}$ \\
ZM & $175,2 \pm 128,4$ & $70,7-376,7$ & 73 \\
ZA & $207,0 \pm 86,4$ & $114,9-334,3$ & 42 \\
ZB & $145,8 \pm 171,7$ & $6,6-442,7$ & 118 \\
ZM & $\mathrm{Mn}$ \\
ZA & $42,8 \pm 21,8$ & $21,6-65,8$ & 51 \\
ZB & $71,6 \pm 40,3$ & $14,8-126,2$ & 56 \\
ZM & $30,6 \pm 16,6$ & $3,4-46,4$ & 54 \\
ZA & $22,9 \pm 9,4$ & $7,7-32,7$ & 41 \\
\hline
\end{tabular}

1)ZB: <1.000 msnm; ZM: 1.000-2.000 msnm; ZA: >2.000 msnm. pastoreaban allí (Figura 1), lo que indica que frente a variaciones en el contenido de $\mathrm{Cu}$, Fe y Mn, la actividad enzimática en el animal será diferente, reflejando las diferencias en el consumo de ellos. La actividad de SOD es dependiente de la concentración de $\mathrm{Cu}$ en la dieta del animal (Xin et al., 1991; Arthington et al., 1996; Lauridsen et al., 1999); pero, no se ha reportado, en la literatura consultada, que la actividad de esta enzima en el bovino estuviera asociada con la concentración de Fe y Mn; sin embargo, se ha descrito que hay isoenzimas SOD Fe- y Mn-dependientes (Murray et al., 2000).

Se observó una tendencia hacia una correlación inversa para la actividad de SOD con la concentración de Zn (Figura 1). Pese a lo anterior, se ha reportado que la actividad de la SOD puede estar relacionada con el consumo de $\mathrm{Zn}$ en la dieta, habiéndose descrito que hay isoenzimas SOD dependientes de Zn (Murray et al., 2000). Por lo anterior, en rumiantes hay presencia de otras formas de SOD, dependientes de minerales diferentes del $\mathrm{Cu}$, lo que indicaría que la determinación de la actividad sanguínea de SOD no solo está relacionada con el balance metabólico nutricional del $\mathrm{Cu}$, sino de otros como $\mathrm{Fe}$ y $\mathrm{Mn}$, lo que requiere investigaciones posteriores.

El promedio y desviación estándar para la actividad de GSH-Px, en los animales objeto del estudio, fue $389 \pm 184 \mathrm{U} \mathrm{g}^{-1} \mathrm{Hb}$, con un rango entre $57 \mathrm{y}$ $936 \mathrm{U} \mathrm{g}^{-1} \mathrm{Hb}$ (Cuadro 2). Los valores hallados en este estudio para GSH-Px son similares a los encontrados por Ceballos et al. (2003), pero trabajos realizados en otros países revelan actividades más bajas, ubicándose en los rangos marginales, según los valores referenciales (Ceballos et al., 1998a; Wittwer et al., 2002).

La dieta de las novillas en este estudio estaba basada en pastoreo, por lo tanto, es directa la repercusión que ejerce la composición del forraje sobre el balance metabólico nutricional de los animales. Así, se ha señalado que la concentración de Se, en el forraje, guarda una relación directa con la actividad sanguínea de GSH-Px en bovinos a pastoreo (Ceballos et al., 1999). Por lo anterior, la actividad de GSH-Px en sangre de novillas, mantenidas en sistemas pastoriles, proporcionará un acercamiento al balance metabólico de Se en el animal.

En este estudio se encontraron animales con actividades deficitarias, bajas o marginales (Figura 2), compatibles con una deficiencia metabólica nutricional de Se. El promedio más bajo para la actividad de GSHPx se encontró en la zona alta (Cuadro 2). Otros estudios revelan que los bajos niveles de Se, tanto en suelo como 
en forraje de zonas altas, pueden ser debidos a factores edáficos propios de la zona, a la alta pluviosidad que causa la lixiviación y la dilución del mineral, y a un rápido crecimiento de ciertas especies forrajeras, que captan el mineral, evitando su disponibilidad en los forrajes que consumen los animales en pastoreo (Underwood \& Suttle, 1999).

Asimismo, teniéndose en cuenta que las fincas de zona alta se encuentran en una región con influencia volcánica, debido a la cercanía con el volcán "Nevado del Ruiz", esta zona puede tener altas concentraciones de azufre (S) por el origen del suelo y por las emanaciones de ceniza volcánica y presencia de aguas termales subterráneas, entre otras. El S es un mineral molecularmente similar al Se, presentándose una interacción antagónica entre ambos; así, se han repor- tado estudios, en los cuales se ha observado que el incremento en el consumo de S disminuye, en forma lineal, la concentración de Se en el animal (Ivancic Junior \& Weiss, 2001). En este estudio no se verificó cuál era el contenido de S en suelos de zonas altas; pero, por su origen volcánico, es presumible que posean una alta concentración para este mineral (Malagón et al., 1995) y, en consecuencia, en los forrajes que crecen allí. Además, el S posee la misma ruta de absorción en los rumiantes que los selenitos (Underwood \& Suttle, 1999), principal fuente de Se en la mayoría de las sales mineralizadas en Colombia. También se ha señalado que el exceso de $\mathrm{S}$ disminuye la absorción de Se en bovinos (Underwood \& Suttle, 1999; Ivancic Junior \& Weiss, 2001). Otro factor a tenerse en cuenta es que las fincas, en esa zona, se caracterizan por ser explotaciones
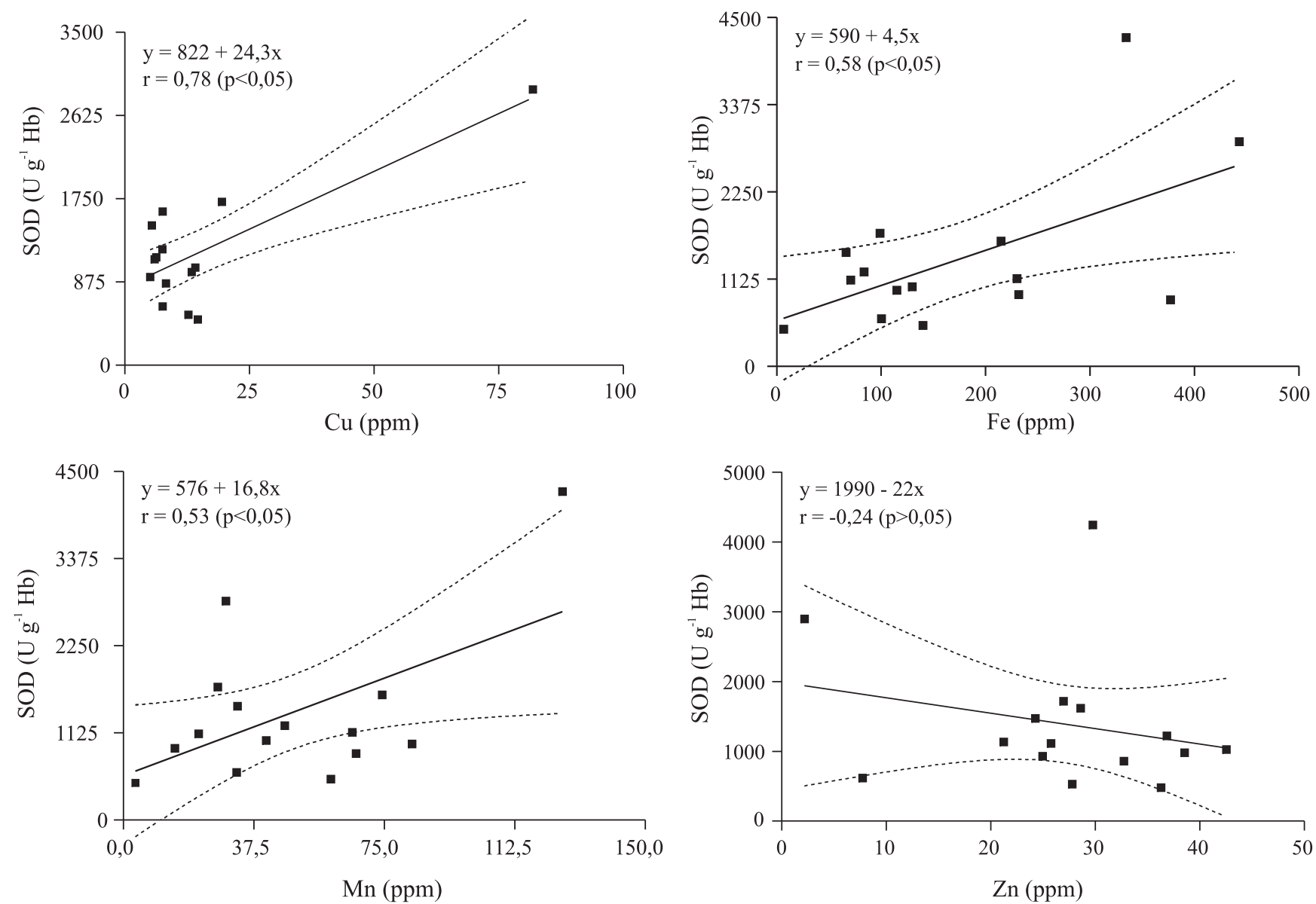

Figura 1. Línea de regresión y correlación entre la concentración de Cu, Fe, Mn y Zn en forrajes y la actividad sanguínea de superóxido dismutasa (SOD), en novillas mantenidas bajo condiciones de pastoreo. 


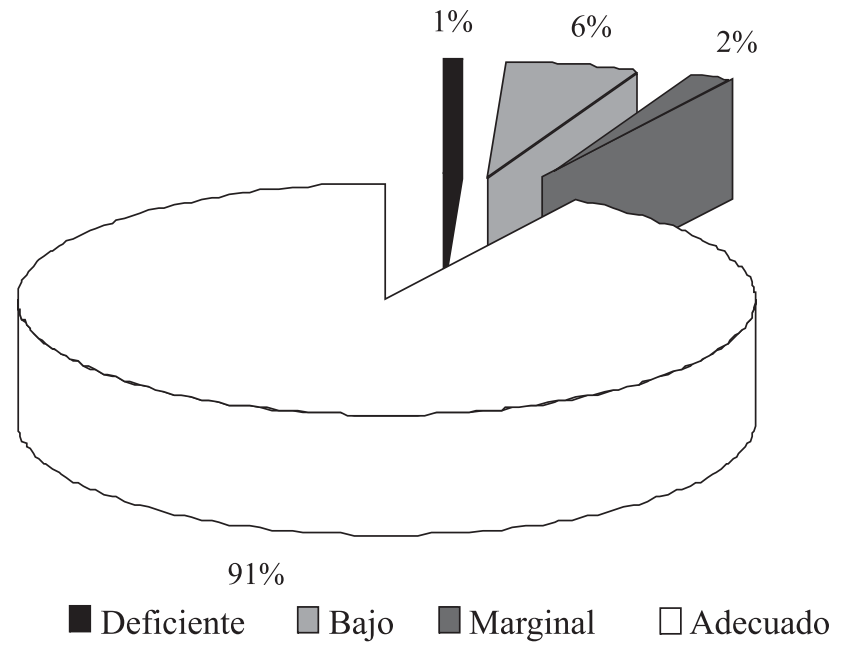

Figura 2. Frecuencia de novillas a pastoreo, con actividad sanguínea de GSH-Px deficiente ( $<60 \mathrm{U} \mathrm{g}^{-1} \mathrm{Hb}$ ), baja (60-100 U g $\left.{ }^{-1} \mathrm{Hb}\right)$, marginal (101-130 $\mathrm{Ug}^{-1} \mathrm{Hb}$ ) y adecuada (>130 U g ${ }^{-1} \mathrm{Hb}$ ), en 15 rebaños lecheros de Caldas, Colombia.

intensivas altamente dependientes de insumos agrícolas, por lo que la aplicación de fertilizantes fosforados también puede disminuir la concentración edáfica de Se (Underwood \& Suttle, 1999).

\section{Conclusiones}

1. La actividad sanguínea de SOD, en bovinos a pastoreo, depende de la concentración de $\mathrm{Cu}$, Fe y Mn en la ración y puede utilizarse como marcador biológico del metabolismo de estos minerales.

2. En Caldas, Colombia, se presentan animales con valores de GSH-Px compatibles con una deficiencia baja/marginal de Se en la dieta.

3. La actividad de GSH-Px varía según la ubicación geográfica de la explotación y se observa la mayor frecuencia de individuos con valores alterados en zonas altas (>2.000 msnm).

4. La suplementación con oligoelementos, en novillas a pastoreo, debe ajustarse según la composición mineral de los forrajes consumidos.

\section{Agradecimientos}

A los propietarios y administradores de los predios donde fue realizado este trabajo; a la Vicerrectoría de Investigaciones y Postgrados de la Universidad de Caldas; a la empresa Proagro Ltda.

\section{Referencias}

ARTHINGTON, J.D.; CORAH, L.R.; BLECHA, F. The effect of molybdenum-induced copper deficiency on acute-phase protein concentrations, superoxide dismutase activity, leukocyte numbers, and lymphocyte proliferation in beef heifers inoculated with bovine herpesvirus. Journal of Animal Science, v.74, p.211-217, 1996.

AWADEH, F.T.; KINCAID, L.; JOHNSON, K.A. Effect of level and source of dietary selenium on concentrations of thyroid hormones and immunoglobulins in beef cows and calves. Journal of Animal Science, v.76, p.1204-1215, 1998.

CAMPBELL, M.H.; MILLER, J.K. Effect of supplemental dietary vitamin $\mathrm{E}$ and zinc on reproductive performance of dairy cows and heifers fed excess iron. Journal of Dairy Science, v.81, p.26932699, 1998.

CEBALlOS, A.; CORREA, H.; LOAIZA J.; VILLA, N.A. Evaluación del balance metabólico nutricional de selenio, cobre y zinc de rebaños lecheros en Manizales, Colombia. Revista Colombiana de Ciencias Pecuarias, v.16, p.19-25, 2003.

CEBALLOS, A.; WITTWER F.G.; ANDAUR, M.; BÖHMWALD, H.L. Actividad sanguínea de glutatión peroxidasa y superóxido dismutasa en equinos del sur de Chile mantenidos en pastoreo. Archivos de Medicina Veterinaria, v.30, p.339-340, 1998b.

CEBAllos, A.; WITTWER, F.G.; CONTRERAS, P.A.; BÖHMWALD, H. Actividad sanguínea de glutatión peroxidasa en rebaños lecheros a pastoreo: variación según edad y época del año. Archivos de Medicina Veterinaria, v.30, p.13-22, 1998a.

CEBALLOS, A.; WITTWER, F.G.; CONTRERAS, P.A.; QUIROZ, E.; BÖHMWALD, H.L. Actividad de glutatión peroxidasa en bovinos lecheros a pastoreo correlacionada con la concentración sanguínea y plasmática de selenio. Pesquisa Agropecuária Brasileira, v.34, p.2331-2338, 1999.

DANIEL, W.W. Bioestadística, base para el análisis de las ciencias de la salud. 4.ed. México, DF: Limusa Wiley, 2002. 755p.

DARGATZ, D.A.; ROSS, P.F. Blood selenium concentrations in cows and heifers on 253 cow-calf operations in 18 states. Journal of Animal Science, v.74, p.2891-2895, 1996.

HAMBIDGE, M. Biomarkers of trace mineral intake and status. Journal of Nutrition, v.133, p.948S-955S, 2003.

HERDT, T.H. Variability characteristics and test selection in herdlevel nutritional and metabolic profile testing. Veterinary Clinics of North America: Food Animal Practice, v.16, p.387-403, 2000.

IVANCIC JUNIOR, J.; WEISS, W.P. Effect of dietary sulfur and selenium concentrations on selenium balance of lactating Holstein cows. Journal of Dairy Science, v.84, p.225-232, 2001.

KINCAID, R.L. The biological basis for selenium requirements of animals. Professional Animal Scientist, v.11, p.26-29, 1995.

LAREDO, M.A.; CUESTA, A. Tabla de contenido nutricional de pastos y forrajes de Colombia. 2.ed. Bogotá, DC: Instituto Colombiano Agropecuario, 1987. 80p.

LAURIDSEN, C.; NIELSEN, J.H.; HENCKEL, P.; SORENSEN, M.T. Antioxidative and oxidative status in muscles of pigs fed rapeseed 
oil, vitamin E, and copper. Journal of Animal Science, v.77, p.105115, 1999.

MALAGON, D.; PULIDO, C.; LLINAS, R.D.; CHAMORRO, C. Suelos de Colombia. Bogotá, DC: Instituto Geográfico Agustín Codazzi, 1995. 632p.

MEE, J.F.; ROGERS, A.M. Relevance of iodine, selenium, copper and cobalt deficiencies on irish cattle farms. Irish Veterinary Journal, v.49, p.529-537, 1996.

MILLER, J.K.; BRZEZINSKA-SLEBODZINSKA, E.; MADSEN, F.C. Oxidative stress, antioxidants, and animal function. Journal of Dairy Science, v.76, p.2812-2823, 1993.

MURRAY, R.K.; GRANNER, D.K.; MAYES, P.A.; RODWELL, V.W. Harper's biochemistry. $25^{\text {th }}$ ed. Stamford: Appleton \& Lange, 2000. 927p.

PAVLATA, L.; ILLEK, J.; PECHOVA, A.; MATEJISEK, M. Selenium status of cattle in the Czech Republic. Acta Veterinaria Brunensis, v.71, p.3-8, 2002.

SCALETTI, R.W.; TRAMMELL, D.S.; SMITH, B.A.; HARMON, R.J. Role of dietary copper in enhancing resistance to Escherichia coli mastitis. Journal of Dairy Science, v.86, p.1240-1249, 2003.
UNDERWOOD, E.J.; SUTTLE, N.F. The mineral nutrition of livestock. $3^{\text {rd }}$ ed. Wallingford, UK: CABI Publishing, 1999. 624p.

WICHTEL, J.J. A review of selenium deficiency in grazing ruminants; part 1: new roles for selenium in ruminant metabolism. New Zealand Veterinary Journal, v.46, p.47-52, 1998.

WICHTEL, J.J.; CRAIGIE, A.L.; FREEMAN, D.A.; VARELAALVAREZ, H.; WILLIAMSON, N.B. Effect of selenium and iodine supplementation on growth rate and on thyroid and somatotropic function in dairy calves at pasture. Journal of Dairy Science, v.79, p.1865-1872, 1996.

WITTWER, F.; ARANEDA, P.; CEBALLOS, A.; CONTRERAS, P.A.; ANDAUR, M.; BÖHMWALD, H. Actividad de glutatión peroxidasa (GSH-Px) en sangre de bovinos a pastoreo de la IX Región, Chile y su relación con la concentración de selenio en el forraje. Archivos de Medicina Veterinaria, v.34, p.49-57, 2002.

XIN, Z.; WATERMAN, D.F.; HEMKEN, R.W.; HARMON, R.J. Effects of copper status on neutrophil function, superoxide dismutase, and copper distribution in steers. Journal of Dairy Science, v.74, p.3078-3085, 1991.

Recibido el 24 de marzo de 2004 e aceptado el 11 de abril de 2005 